\title{
Gewissens-Bisse. Wie Juan Azors Verständnis der Gewissenslagen zu einer am Tierwohl orientierten Praxis anleiten kann
}

\section{Martina Besler}

\section{Die Notwendigkeit einer Tugendethik als Brücke zur Praxis im Kontext der Tierethik}

Innerhalb der Tierethik dominieren im deutschsprachigen Raum bislang normativ-ethische Ansätze, wie anhand aktueller tierethischer Werke nachzuvollziehen ist (z. B. Martin M. Lintner 2017; Michael Rosenberger 2015; Kurt Remele 2016 u. v. m). Doch zeigt sich, dass diese normativen Ansätze trotz ihrer ähnlichen Zielsetzung dennoch oftmals keine entsprechende Veränderung in der Praxis nach sich ziehen. Dies mag viele Gründe haben. So fördern die ökonomischen Rahmenbedingungen und ein gewisser gesellschaftlicher Druck zu besonders kostengünstigen Lebensmitteln durchaus eine Ernährung, durch welche das Wohl des Tieres in den Hintergrund rückt. Welche und in welchem Umfang tierliche Produkte gebraucht werden, ist in starkem $\mathrm{Maß}$ den individuellen menschlichen Entscheidungen überlassen, da es in der westlichen, christlich geprägten Kultur kein staatliches oder kirchliches Gesetz gibt, welches diesen Konsum und die damit einhergehenden Praktiken ausreichend regelt. Die aktuellen Tierschutzgesetze (z. B. das Tierschutzgesetz in Deutschland [Bundesministerium der Justiz und für Verbraucherschutz/Bundesamt für Justiz 1972]) sind teils vage, teils unzureichend formuliert und können leicht umgangen werden, wie z. B. die bestehende Praxis von Massentierhaltung zeigt (Heinrich-Böll-Stiftung et al. 22018, 24-25). Diese Diskrepanz, d. h. das Vorhandensein von Sachwissen, ohne praktische Konsequenzen zur Folge zu haben, bezeichnet Markus Vogt im Kontext der Ökologie als den „Graben zwischen Wissen und Verantwortung“ (Markus Vogt 2019, 10).

Eine mangelnde Umsetzung von Einsichten im Kontext der Tierethik fällt umso mehr auf, als die in den normativen Ethiken genannten Ziele Gesundheit für den Menschen und Wohl für die Tiere - in unserer Gesellschaft durchaus akzeptierte Zielvorstellungen sind. Zumindest zeigt die Existenz von teilweise stärker werdenden Gruppen wie Vegetarier*innen und Veganer*innen (Gert B. M. Mensink et al. 2016, 2-15), welche neue 
Angebote im Lebensmittel- und Gastronomiegewerbe erzeugen, ebenso wie Tierschützer*innen, welche die Frage nach dem Tragen von Pelz und Skandale um falsch deklarierte Kunstpelze in der Gesellschaft präsent halten (Joana Ciraci 2019), dass Gesundheit und Tierwohl als Leitvorstellungen akzeptiert sind.

Doch nicht nur in der Gesellschaft, auch unter Christ"innen lässt sich dasselbe Phänomen feststellen. Unter dem religiösen Anspruch des sorgsamen Umgangs mit der Schöpfung steht die ethische Frage nach dem Umgang mit dem Konsum tierlicher Produkte auch für Christinnen und Christen an. Der Schutz tierlicher Lebewesen, insbesondere von Kühen, Schweinen, Puten, Hühnern und Fischen, findet auch in der Katholischen Kirche immer noch viel zu wenig Beachtung. Eine klare Positionierung dazu vermisst man auf Ämterebene, an zahlreichen Universitäten sowie allgemein unter Christen und Christinnen. Ein sehr prominenter Anstoß von heute blieb in vielen christlichen Familien, in Pfarrhäusern und in Ordensgemeinschaften unbeachtet. Es handelt sich dabei um die Enzyklika Laudato si' von Papst Franziskus. Trotz vieler guter Worte des Papstes in die richtige Richtung, hin zu einer größeren Wertschätzung unserer Mitgeschöpfe (Nr. 69), hat sich in den christlichen Familien, in Pfarrhäusern und in Ordensgemeinschaften in der Praxis kaum etwas geändert, und es kommen weiterhin fast täglich mehr tote Stücke von Gottes Schöpfung in Form von Wurst und Fleisch auf den Tisch (Gabriele Höfling 2016), als im Sinne des Tierwohls und auch im Sinne einer Abwägung von Tier- und Menschenwohl zu rechtfertigen sind. Dabei verweisen die normativen Ansätze der Tierethik eindeutig auf das hier missachtete Wissen von der Leidensfähigkeit vieler Tiere, den Mangel an artgerechter Tierhaltung, die tierliche als auch menschliche Gesundheit und letztendlich den Wert des Lebens. Es scheint, als verorteten auch zahlreiche Christ"innen ihre persönliche Haltung nicht im großen Ganzen der Schöpfung Gottes und würden dem biblischen Anspruch auf Verantwortungsübernahme für die Schöpfung Gottes nicht ausreichend gerecht werden.

Es legt sich daher die Schlussfolgerung nahe, dass normative Ansätze oftmals alleine nicht ausreichen, um eine Veränderung des Verhaltens zu veranlassen. Aus diesem Grund möchte dieser Beitrag den Blick auf die Bedeutung der Tugendethik als einer Form der Tierethik lenken, die in stärkerem Maß die praktische Umsetzung in den Blick nimmt. ${ }^{1}$ Da der Um-

$1 \mathrm{Zu}$ den unterschiedlichen Ansätzen in der Tierethik, z. B. Tugendethik, Mitleidsethik, Gerechtigkeitstheorien und viele mehr, vgl. Johann S. Ach/Dagmar Borchers [Hg.] 2018. 
fang dieser Arbeit keine Entfaltung des Themas der Tugendethik erlaubt, wird sich die Darstellung inhaltlich auf den Umgang mit Tierprodukten aus der Perspektive des Tierwohls und systematisch auf die Frage nach der Verbindung von Theorie und Praxis, d. h. auf das Zusammenspiel von Einsicht, konkreter Handlung und dem Erwerb von (Tugend-)Haltungen beschränken müssen. Dabei muss außerdem vorausgesetzt werden, dass mit der Perspektive des Tierwohls von vorneherein eine gewisse Komplexität verbunden ist, weil unterschiedliche ethische Ansätze zu unterschiedlichen Gewichtungen des Tierwohls gelangen und daher innerhalb der vorgegebenen Zielsetzung bereits von einem gewissen Spektrum an ethisch begründbaren Positionen auszugehen ist (Johann S. Ach/Dagmar Borchers [Hg.] 2018). Ein Aspekt, der die Komplexität zusätzlich erhöht, ist der mögliche persönliche Wechsel zwischen begründbaren Positionen.

Bei dieser Pluralität möglicher Positionen setzt nun dieser Beitrag an, indem er die Frage verfolgt, welche Hilfestellung es im Bereich der theologischen Ethik für die Ausbildung persönlicher tugendethischer Haltungen geben könnte, die in engerer Verbindung mit der Praxis stehen und zur Umsetzung eigener Einsichten verhelfen. Dazu wird auf das klassische Anliegen der Gewissensbildung rekurriert und eine Methode vorgestellt, die in Anlehnung an Juan Azor (+ 1603), den ersten Autor eines moraltheologischen Lehrbuchs der Frühen Neuzeit, entwickelt wird. Der Rückgriff auf derartige Quellen zur Lösung aktueller ethischer Fragestellungen scheint ein ungewöhnlicher Weg zu sein, doch stellen die Ausführungen von Juan Azor zum Gewissen in seinen Institutiones morales (Juan Azor 1600) einen wertvollen Beitrag für eine Standortbestimmung des Handelnden in Fragen der Umsetzung dar, wie im Folgenden zu zeigen sein wird.

Die Gewissenslehre bildet bei Azor den Mittelpunkt seines gesamten Werkes (Johann Theiner 1970, 269) und insbesondere seine Entfaltung der Lehre von den Gewissensfällen kann für praktische Fragen der Gewissenshermeneutik und gelebter ethischer Praxis noch heute impulsgebend sein. Dass ein tugendethischer Zugang seinen Ausgangspunkt beim Gewissen nimmt, ist deshalb wichtig, weil der Grad der Sensibilität des Gewissens in enger Verbindung zur Ausbildung tugendethischer Haltungen steht (Klaus Demmer 1985, 58). Azor zeigt in seinen Ausführungen, dass sich unterschiedliche Menschen in unterschiedlichen „Gewissenslagen“ befinden. Ein Bewusstsein für diese zu gewinnen, kann daher für Menschen einen ersten Schritt der Selbsterkenntnis darstellen und zur Entwicklung einer bewussten und verantworteten Haltung veranlassen. Daher verstehen sich die nachfolgenden Ausführungen vornehmlich als ein Beitrag zu einem moralpädagogischen Aspekt der Tugendethik. 


\section{Die Reflexion der „Gewissenslagen" bei Juan Azor}

Die oben beschriebene Spannung zwischen ethisch-normativem Wissen und verantwortlichem Handeln lässt erkennen, dass das Verständnis des Gewissens (lat. conscientia) im Sinne des Thomas von Aquin (+1274), nämlich als konkretes Urteil der praktischen Vernunft (Eberhard Schockenhoff 1990, 78), voraussetzungsreich ist, was die Umsetzung angeht: Thomas geht davon aus, dass eine gegebene Einsicht auch ein praktisches Urteil zur Folge hat und das praktische Gewissen die Übereinstimmung des Handelns mit diesem Urteil bewertet bzw. zur Umsetzung auffordert. Nun gibt die Realität im Hinblick auf den Umgang mit tierlichen Produkten zu erkennen, dass es zwischen allgemeiner Einsicht und praktischem, unmittelbar handlungsanleitendem Urteil ein paar Hindernisse gibt. Während Hintergründe der Produktion von tierlichen Produkten oftmals bekannt sind und aus christlicher Perspektive auch eine allgemeine Anerkennung von Tieren als Mitgeschöpfen gegeben ist, folgen daraus keineswegs notwendigerweise ausreichend Konsequenzen im konkreten Handeln. Es scheint beispielsweise, dass das Wissen über die Umstände der Massentierhaltung im persönlichen ethischen Urteil oftmals zu keiner Veränderung der persönlichen Praxis führt, also auch für das Gewissensurteil nicht relevant erscheint. Ein Blick auf die Lehre von den „Gewissenslagen“ bei Juan Azor hilft zu verstehen, wie es möglich ist, dass offensichtlich Wissen und Einsichten übergangen oder verdrängt werden und es zu keiner Änderung im Lebensstil kommt, d. h. auch diesbezüglich keine tugendethische Haltung aufgebaut wird.

Juan Azors Ausführungen sind in der Lehre der katholischen Moraltheologie im 16. und 17. Jahrhundert zu verorten, welche von einem Umbruch von einer stärker grundlegungsorientierten Reflexion im Mittelalter hin zur Kasuistik charakterisiert ist, mit welcher ein Verständnis des Gewissens im Sinne eines Gesetzesgehorsams verbunden wird (Martin Grabmann 1933, 180). In seinem Lehrbuch spricht Azor sowohl dem Gesetz eine wichtige Rolle zu, besonders bezogen auf das Gemeinwohl, aber hebt auch - insbesondere in privaten Angelegenheiten - die individuelle Freiheit hervor. Azor kann somit noch nicht der Linie des zuvor skizzierten Legalismus der Kasuistik zugerechnet werden. ${ }^{2}$ Sein Interesse am Gewissen

2 Azors Verständnis von Gewissen weist sogar strukturelle Ähnlichkeiten zu einer Vorstellung vom Gewissen auf, die wir heute in Anlehnung an Alfons Auer als „autonom im christlichen Kontext“ (Wolfgang Nethöfel 1987, 79-124) bezeichnen würden. In den Institutiones morales wird keine moralische Entscheidung nur unter Berufung auf Gott gefällt, sondern sie erfolgt immer in Verbindung mit der Ver- 
ist weniger ein theoretisches, wie es sich in den Diskussionen vieler mittelalterlicher Autoren zeigt; vielmehr geht es ihm primär um die praktische Bedeutung des Gewissens. An diesen Fokus schließen auch die folgenden Überlegungen an, die Azors „Gewissenslagen“ zunächst darstellen und dann zu aktualisieren versuchen.

Azor entfaltet verschiedene Phänomene des Gewissens, von denen einige bei Thomas von Aquin noch nicht reflektiert wurden und auch nicht wie bei ihm den Fokus auf das Gewissensurteil richten. Die unterschiedlichen Gewissensphänomene interpretiert Azor eher im Sinne von „Gewissenslagen“. Dazu gehören bei ihm neben dem rechten Gewissen (conscientia recta) und dem irrenden Gewissen (conscientia errans) das meinende Gewissen (conscientia opinans), das zweifelnde Gewissen (conscientia dubia) und das skrupulöse Gewissen (conscientia scrupulosa) (Juan Azor 1600, lib. 2, cap. 8).

Das rechte Gewissen ist leicht erklärt, denn es ist das Gewissen, das Gutes vorschreibt und entsprechende Handlungen auch als wirklich gut begreift. Das irrende Gewissen wird definiert als Akt der Vernunft, welcher vorschreibt, etwas zu tun, was sich den Anschein des Guten gibt, obwohl es etwas Schlechtes ist. Das Gewissen irrt also in der Deutung der Handlung (Juan Azor 1600, lib. 2, cap. 8, q. 2). Das meinende Gewissen ist gleichbedeutend mit dem probablen Gewissen. Hierbei geht es darum, innerhalb mehrerer vernünftig begründbarer Positionen eine für sich als handlungsleitend auszuwählen; es handelt sich somit nicht um willkürliche Meinungen (Juan Azor 1600, lib. 2, cap. 9). Das zweifelnde Gewissen bezeichnet die

nunft, die auch bei Auer die tragende Funktion einnimmt (Alfons Auer 1971, 17). Beide Autoren greifen für ihre Argumentation auf Thomas von Aquin zurück (Alfons Auer 1971, 17/Juan Azor 1600, lib. 2, cap. 8, q. 1).

Auer beschreibt in seinem Buch „Autonome Moral und christlicher Glaube“ den Zusammenhang zwischen Rechtsphilosophie und Moraltheologie. Moralische Gewissheit bzw. die Gewinnung des Rechts ist beides nur in deren Komplexität möglich, was für die Moral eine Bündelung der Probabilitäten bedeutet und für das Recht das Zusammenkommen verschiedener Elemente, wie soziale Strukturen, Sitten und Bräuche, Gerechtigkeit und einiges mehr (Alfons Auer 1971, 49-52; Heinrich Henkel 1964, 419-421). Und gerade diese Sittlichkeit, die sich hierbei ausbildet, ist autonom (d. h. gestützt auf die Vernunft) zu entwickeln. Das bedeutet nicht, dass dies gegen den christlichen Glauben geschieht, sondern im Vollzug der religiösen Verbundenheit mit Gott (Alfons Auer 1971, 178). Dieses Verständnis von Sittlichkeit bzw. des Gewissens ist auch bei Azor zu entdecken. Besonders deutlich wird das in folgendem Zitat Azors: „Animadvertendum tamen est, non ideo semper opinionem esse eligendam, quia magis pia esse videatur: nam in hoc non sufficit sola pietatis species, si ratio, vel maior auctoritas repugnet" (Juan Azor 1600, lib. 2, cap. 15). 
Situation, dass nicht ersichtlich ist, was das Gute ist; die Person mit einem zweifelnden Gewissen gelangt aufgrund des Zweifelns nicht zum Handeln. In diesem Fall rät Azor dem zweifelnden Menschen dazu, einer sicheren Option zu folgen. Insofern vertritt er keinen allgemeinen, sondern einen gruppenspezifischen Tutiorismus, doch selbst diesen nicht in rigoroser Weise: auch in diesem Fall hält er es für legitim - hierin zeigt sich der Einfluss der probabilistischen Position des Dominikaners Bartolomé de Medina (+ 1580) -, die sichere Option zu verlassen und einer wahrscheinlichen Meinung zu folgen, wenn es sich dabei beispielsweise um den Rat eines klugen Menschen handelt (Juan Azor 1600, lib. 2, cap. 18, q. 1). Zuletzt behandelt Azor noch das skrupulöse Gewissen. Azor beschreibt es als Unruhe im Geist und verdeutlicht es durch die Metapher kleiner unbedeutender Steinchen im Schuh, die auf Dauer stark quälen können. Er bezeichnet damit Entscheidungen, die leichtfertig getroffen werden, bei denen sich die Handelnden daher öfter umentscheiden und die am Ende zu Bedenken in Bezug auf alle möglichen Entscheidungen führen (Juan Azor 1600, lib. 2, cap. 10, q. 1).

Es wird deutlich, dass auch zu Azors Zeit die Komplexität von Umständen ein Spektrum von möglichen sittlichen Urteilen hervorrufen konnte. Dabei erfordern nach Azor unterschiedliche persönliche Situationen auch unterschiedliche Lösungsansätze, um zu einer guten, konkreten Entscheidung zu gelangen. Azor verschrieb sich daher keinem der im 17. Jahrhundert diskutierten Moralsysteme eines radikalen Tutiorismus, Probabilismus oder Laxismus gänzlich. Stattdessen sucht er für die konkret betroffenen Menschen einen gangbaren Weg. Dabei ist für seine Position die anthropologische Grundeinsicht ausschlaggebend, dass niemand fehlerfrei ist (Juan Azor 1600, lib. 2, cap. 10). Ziel ist es, Selbstständigkeit und Autonomie im eigenen Urteil zu verstärken und Hemmnisse beim Entscheiden und gewissensgemäßen Handeln zu reduzieren. Wie die von Juan Azor entwickelten Unterscheidungen als moralpädagogische Elemente im Kontext des Umgangs mit tierlichen Produkten zur Selbstreflexion ebenso wie im Sinne einer Hilfestellung für andere eingesetzt werden könnten, soll nun im Folgenden dargestellt werden. Dabei ist es interessant zu sehen, dass die Hindernisse für die Ausbildung einer handlungswirksamen Position bei Azor de facto nicht nur die kognitive, sondern auch die Gemütsebene in den Blick nehmen. ${ }^{3}$

3 Für einen zeitgemäßen Zugang zum Verhältnis von kognitiven und emotionalen Teilen der Persönlichkeit im Hinblick auf ethische Entscheidungsfindung vgl. Rupert Grill 2019. 


\section{Persönliche Gewissensentwicklung auf der Grundlage der Gewissenslagen} Azors

Dieses Bemühen, einen Weg persönlich tragbarer und verantwortbarer Freiheit zu suchen, kann man in moraltheologischer Umdeutung eines von Karl Rahner gebrauchten Begriffs veranschaulichen, der gleichsam eine Brücke von Azor in die neuere Zeit und zur aktuellen Tierethik erlaubt. Karl Rahner spricht im Kontext der Ekklesiologie von einem „Tutiorismus des Wagnisses“ (Karl Rahner 1995, 313-316). Er möchte mit diesem Begriff das Handeln der Kirche zu einem Fortschritt ermutigen: die Kirche solle in der Lösung eines Problems nicht nur so weit gehen, wie es von der Situation erzwungen werde, sondern sie solle sich fragen, wie weit sie unter Ausnützung aller theologischen und pastoralen Möglichkeiten gehen dürfe; die Lage des Reiches Gottes erfordere es nämlich, dass die Kirche das Äußerste wage, um vor dem Anspruch Gottes zu bestehen. Das Wagnis wird hierbei zum Prinzip; es wird in einem Zweifelsfall als die dem Anspruch Gottes gemäßere Option angesehen. Es geht also darum, eine radikalere, eine mutigere, eine mehr wagende und somit auch eine neue Lösung für die Fragen der jeweiligen Zeit zu finden. In analoger Weise könnte man auch im Hinblick auf eine Steigerung des Tierwohls von einem moralpädagogischen „Tutiorismus des Wagnisses“ sprechen. Dieser würde etwa folgendermaßen lauten: Jede Person, besonders auch die Christ"innen, sollen in Fragen des Tierwohls nicht nur so weit gehen, wie es von der Situation erzwungen wird, sondern sie sollen sich fragen, wie weit sie unter Ausnützung aller persönlichen Möglichkeiten gehen können, weil es die Verantwortung für die Schöpfung verlangt, Bequemlichkeiten zurückzulassen und angesichts der von Gott dem Menschen übertragenen Verantwortung vor dem Anspruch des eigenen Gewissens zu bestehen. Dieser Tutiorismus des praktischen Wagnisses im Sinne des Tierwohls setzt freilich bereits voraus, dass das Tierwohl als Norm des persönlichen Gewissens zuvor auch ethisch gerechtfertigt wurde. ${ }^{4}$

In Anlehnung an Azors Herangehensweise an die Themen seiner $\mathrm{Zeit}^{5}$ werden daher im Folgenden drei Schritte dargestellt, wie die Trägheit überwunden werden kann, die das Zustandekommen und die Umsetzung

4 In diesem Rahmen kann aufgrund des beschränkten Platzes der Nachweis nicht geführt werden, doch wird auf die o. g. Literatur verwiesen.

5 Azor beschäftigt sich in den Institutiones morales in Verbindung mit dem Gewissen mit aktuellen Themen seiner Zeit. Diese waren z. B. im Kontext des Konzils von Trient (1545-1563) die Gültigkeit und Verbindlichkeit der Ehe (Juan Azor 1600, lib. 2 , cap. 18 , q. 5 bis cap. 19 , q. 4). 
eines Gewissensurteils behindert. Der Fokus liegt dabei auf dem Konsum tierlicher Produkte und insbesondere der Ernährung, kann aber natürlich auch auf andere Konsumgüter übertragen werden. Ich möchte diese Herangehensweise im Dreierschritt Kognition - Emotion - Habitualisierung erläutern.

\subsection{Gewissenslagen als Hilfe zur Analyse der kognitiven Ebene: vom (mangelnden) Engagement, ethisches Wissen zu sammeln und zu verbreiten}

Jeder Mensch steht in der Pflicht, sich über die Auswirkungen des gegenwärtigen Konsums tierlicher Produkte zu informieren und sich persönlich darüber Gedanken zu machen. Diese Pflicht erwächst alleine schon aus der Fähigkeit des Menschen zum Mitleiden mit anderen leidensfähigen Tieren und seiner ethischen Verantwortung, „hinter die Kulissen zu schauen“, d. h. nachzudenken, welche Folgen das eigene Handeln hat. Diese Folgen haben zahlreiche unterschiedliche Aspekte: einerseits das Leiden und das Töten der Tiere, die wir essen, andererseits das Leiden der Menschen, die die Tiere töten müssen. Weiterhin zählen dazu die Folgen des übermäßigen Fleischgenusses in Form diverser Krankheiten, wie z. B. Diabetes, Darmkrebs etc. (José L. Domingo/Martí Nadal 2017, 256-261), der Klimawandel durch die gegenwärtige industrielle Form der Nutztierhaltung (Redaktion Fleischwirtschaft 2018, 30-31) und, noch weitergedacht, das mangelnde Grundnahrungsangebot in Nicht-Industrieländern, deren Felder für Futtermittel zur späteren Fleischproduktion belegt sind (Philip Lymbery 2015). Hierbei können die gängigen Forschungen zur Tierethik herangezogen werden. Natürlich wäre es aber unrealistisch zu fordern, dass jede*r sich mit allen theoretischen Ansätzen oder mit allen Details auseinandersetzen solle. Eine ausgeweitete Informationspflicht auf Lebensmitteln und entsprechende Informationsprogramme im kirchlichen Kontext und in Schulen wären deshalb eine gute Unterstützung für die persönliche Entscheidungsfindung.

Über diese generelle Pflicht zur Informationsbeschaffung über die Formen des gegenwärtigen Fleischkonsums hinaus kommt im Hinblick auf die konkrete Entscheidungspraxis das meinende oder probable Gewissen zum Tragen. Nach Azor kann in einem Fall, in welchem es mehrere mögliche Lösungswege gibt, gewählt werden, welchem man folgt. Allerdings trifft dies nur zu, wenn Zweifel ausgeschlossen sind (Juan Azor 1600, lib. 2, cap. 12). Ein Beispiel für eine solche Situation könnte die Frage sein, ob man aufgrund der gesundheitlichen Folgen den Konsum tierlicher Produkte einschränken soll. Azor würde in einem solchen Fall für eine Ent- 
scheidung freie Hand geben, da es verschiedene Meinungen zu diesem Thema gibt und auf jeder Seite gute Gründe stehen. Allerdings würde Azor darauf hinweisen, dass darauf geachtet werden muss, wessen Meinung als ausschlaggebend gesehen wird: Die Autorität und die Stellung der Person, deren Meinung befolgt wird, sind hierbei wichtig (Juan Azor 1600, lib. 2, cap. 14). Heute würde im Kontext ethischer Argumentation statt eines Autoritätsarguments eher die Sachkenntnis im Sinne der wissenschaftlichen Ausgewiesenheit einen Bezugspunkt darstellen.

Ein zweites Beispiel aus dem Bereich der Informationspflicht betrifft die Situation eines irrenden Gewissens. Ein Beispiel dafür wäre der gedankenlose Kauf von Wurstsemmeln in der Mittagspause. Hier zeigt sich die Bedeutung der Wissenskomponente eines Urteils. So ist nach Azor zwar dem irrenden Gewissen zu folgen, aber nur, wenn der Irrtum nicht selbst verschuldet ist (Juan Azor 1600, lib. 2, cap. 8, q. 3-5). Bezogen auf den Konsum tierlicher Produkte würde das bedeuten, dass jemand, der noch nie etwas von industrieller Massentierhaltung gehört hat und auch nicht die Chance hatte, sich darüber zu informieren, sich nicht beim Kauf solcher Produkte schuldig macht. Wer sich jedoch absichtlich vor der Realität blind stellt, muss das selbstverschuldet irrende Gewissen ablegen und so von einer „Sorglosigkeit“ zu einem bewussten Konsum oder Nicht-Konsum übergehen.

Ein weiteres Element im Bereich des ethisch relevanten Wissens, das über die Beschaffung von Information und die Selbstvergewisserung in entsprechenden Situationen hinausführt, ist das Verbreiten dieser Informationen. In analoger Weise zum Austausch von Wissenschaftler*innen über ihre Forschungsergebnisse sollten auch die handelnden Menschen ihr persönliches Wissen bezüglich der Ernährungsweise mit anderen teilen und auf diese Weise Verantwortung für die Weitergabe von ethisch relevantem Wissen übernehmen.

\subsection{Gewissenslagen als Hilfe zur Analyse der emotionalen Ebene: Schlussfolgerungen ziehen und in der Praxis überprüfen}

Wie bereits angesprochen, führt die kognitive Erkenntnis für sich genommen oftmals nicht zu einer Veränderung der Praxis. Grimm und Wild sprechen an dieser Stelle in Bezug auf das Fleischparadox ${ }^{6}$ von einer

6 Darunter versteht man das Fleischessen und damit die Teilnahme an der Praxis der Fleischproduktion inkl. aller damit zusammenhängenden Probleme, obwohl man 
„strukturellen Willensschwäche“ (Herwig Grimm/Markus Wild 2016, 212), die viele Menschen sogar offen zugeben, wenn es um den Konsum tierlicher Produkte geht. Den Grund dafür sehen die genannten Autoren in der Psyche bzw. im sozialen Umfeld (Herwig Grimm/Markus Wild 2016, 210-212). Deshalb ist eine Moralpädagogik in kleinen Schritten erforderlich, die dort ansetzt, wo die einzelne Person die meisten Anknüpfungspunkte hat. Oft ist das Interesse für eines unter den vielen vorher angesprochenen Themen besonders groß: für das Leid der Tiere, die Würde von Tieren oder Menschen oder das Geschöpf an sich, vielleicht für den Klimawandel. Der/die Einzelne sollte sich diesbezüglich klarwerden, welches Argument persönlich die höchste Überzeugungskraft besitzt und dazu motiviert, etwas am eigenen Handeln verändern zu wollen. Dieses Ziel vor Augen, können erste kleine Schritte gesetzt werden. Es geht also nicht etwa sofort um einen gänzlichen Verzicht auf tierliche Produkte, sondern beispielsweise darum, den Versuch zu wagen, den Konsum zu verringern oder bewusst eine Zeit lang auf tierliche Produkte zu verzichten. Dieses Vorgehen fällt einem Menschen, der sehr auf Gewohnheiten bedacht ist, leichter, da nicht sofort der Anspruch einer kompletten Lebensumstellung im Raum steht. Einen solchen „vegetarischen Probemonat“ oder vielleicht sogar auch einmal einen ,veganen Probemonat“ (bzw. Woche) kann dagegen jeder und jede je nach Empfinden öfter ausprobieren.

In dieser Phase des persönlichen Ausprobierens ist es nun möglich, dass es zu einem zweifelnden Gewissen kommt. So kann das Gewissen z. B. im Bezug darauf in Zweifel geraten, ob das tierliche Produkt, ob nun Fleisch oder Käse etc., aus industrieller Massentierhaltung stammt oder nicht. In vielen Restaurants ist dies z. B. nicht gekennzeichnet, aber auch im Supermarkt ist die Haltungsweise der Tiere oft nicht ersichtlich. Nach Azor ist dann nach dem Grundsatz vorzugehen, dass das gewählt werden muss, was sicher mit keiner Sünde verbunden ist (Juan Azor 1600, lib. 2, cap. 18, q. 4). Analog würde das heute bedeuten: was sicher nicht dem informierten Urteil des Gewissens widerspricht. Da industrielle Massentierhaltung zumindest als „strukturelle Sünde“ (Rainer Hagencord) gewertet werden kann (Hilde Regeniter 2017; Interview mit Rainer Hagencord), sind die daraus gewonnenen Produkte ganz abzulehnen und ist eine Alternative dazu zu suchen. Diese Alternative kann sowohl der Einsatz für bessere Praktiken in der Tierhaltung als auch der völlige Verzicht auf tierliche Produkte sein.

gleichzeitig dagegen ist, Tieren Schmerz und Leid zuzufügen, und Mitleid mit diesen hat (Herwig Grimm/Markus Wild 2016, 210-211). 
Wenn nicht erkennbar ist, woher die Produkte stammen, dürften sie nach Azors Auffassung nicht ausgewählt werden. Er würde also vermutlich zu Produkten raten, die aus Produktionen stammen, welche auf das Tierwohl achten. Dass jeder ganz auf tierliche Produkte verzichten muss, würde Azor vermutlich nicht verlangen, da aus seinem Text sehr wohl herauszulesen ist, dass er die Schwäche des Menschen kennt (Juan Azor 1600, lib. 2, cap. 10; cap. 18, q. 5). Er verweist hierbei auf die Gewohnheit der Menschen, die zu einem bestimmten Verhalten führt und in heutigen Analysen mit der Komponente des sozialen Umfeldes verbunden wird (Herwig Grimm/Markus Wild 2016, 212).

Dieser Gewohnheitskomponente ist demnach durch praktisches Ausprobieren entgegenzuwirken, um so alte Gewohnheiten zu unterbrechen; außerdem ist es notwendig, die eigenen Taten und Handlungen zu prüfen und darüber zu reflektieren. Dies ist wichtig, um sich selbst bewusst zu machen, warum man etwas ändern möchte bzw. geändert hat und die gesamtmenschliche, also auch emotionale Reaktion darauf wahrzunehmen. Hierbei kann es helfen, nach einer eigenen Reflexion mit anderen Personen darüber zu sprechen. So ist es möglich, die verschiedensten Denkanstöße für sich selbst einzuordnen und zu bewerten, festzuhalten und neue Praktiken zu bejahen. Bleiben die ersten Versuche, wie z. B. ein „vegetarischer Probemonat", einfach so stehen, ohne eine Reflexion nach sich zu ziehen, verliert sich diese Idee/dieser Versuch sehr schnell im Alltag und hat keine weiteren Folgen für das eigene Leben. Ein gutes Beispiel hierfür sind Neujahrsvorsätze.

\subsection{Gewissenslagen als Hilfe bei der Analyse des Schrittes der Habitualisierung: machbare Handlungen in den Alltag einbauen und weiter erproben}

Hingegen ist es möglich, nach einer Reflexion die neuen Erkenntnisse zumindest stückweise in den Alltag einzubauen. Vielleicht ist es möglich, aus diesem einen „vegetarischen Probemonat“ mehrere hintereinander zu machen, oder aber es wird erkannt, dass die Wurst am Frühstücksteller gar nicht mehr so recht „schmecken“ will - schmecken steht hier in Anführungszeichen, denn der Geschmack der Wurst hat sich nicht verändert, allerdings die Notwendigkeit und die Sichtweise darauf, diese essen zu wollen -, so dass ein völliger Verzicht möglich wird. Wichtig ist hierbei, sich weiterhin das Warum der Handlung und Entscheidung ins Gedächtnis zu rufen. Vergisst man den Grund dahinter, schleichen sich alte Gewohnheiten in Form von sogenannten "Cheatdays" allzu schnell wieder ein. Und von diesen Ausnahmen gerät der Mensch allzu leicht wieder in die Lage 
des „vorgetäuschten Unwissenden“, der die Realität zu (zumindest im Augenblick) seinen Gunsten von sich abschirmt (João Graça et al. 2016, 353-364).

Natürlich ist es schon ein großer Erfolg, dauerhaft die eigene Ernährung in bestimmten Aspekten umzustellen, jedoch ist es ein Irrglaube zu meinen, dass damit die Welt gerettet wäre, der Klimawandel abgewendet würde und alle Kühe und Schweine auf der Erde glücklich wären. Es ist notwendig, wie in allen Bereichen des Lebens, immer weiter an sich zu arbeiten und das immer neu dazu erworbene Wissen in die Tat umzusetzen. Dafür, wie das im Detail aussehen kann, gibt es keine einheitliche Lösung, da man sich immer im Erkenntnisstand der eigenen Zeit befindet und immer wieder neue Gefahren, aber auch Chancen für ein gutes Zusammenleben von allen Lebewesen aufgezeigt werden. Der Mensch hat dabei die Möglichkeit, sich seiner Freiheit entsprechend zu verhalten. Vielleicht ist jetzt die Zeit gekommen, ganz als Vegetarier zu leben? Oder die Zeit für einen „veganen Probemonat“? Überlegungen in diese Richtung würde der „vegantarische Imperativ“ von Kurt Remele unterstützen (Kurt Remele 2016, 148). Oder einen veganen Tag in der Woche? Oder aber auch zu einem verantworteten Konsum tierlicher Produkte, wofür es ebenso Begründungen gibt (Udo Pollmer et al. 2015). Ob das nun im Zeitraum eines Jahres oder in zehn Jahren geschieht, ist erst einmal zweitrangig. Es gibt aber jedenfalls keinen Grund, sich komplett blind gegenüber den Problemen zu stellen oder Entschuldigungen zu suchen. Das große Problem ist, dass der Impuls aus jedem Menschen selbst kommen muss und jeder Einzelne nur für sich persönlich die Gründe kennt, welche ihn/sie dazu antreiben. Und diese Verantwortung kann niemandem abgenommen werden.

$\mathrm{Zu}$ viele unterschiedliche Meinungen aus diversen, vielleicht nicht nachvollziehbaren Richtungen können jedoch auch zu einem skrupulösen Gewissen führen. Am Ende ist man sich nicht mehr sicher, was richtig und was falsch ist, ob es nun erlaubt ist, tierliche Produkte zu essen oder nicht, wieviel davon, woher sie stammen dürfen usw. Die Person zweifelt schlussendlich an allem und bleibt in einem Zustand der Orientierungslosigkeit zurück. In diesem Fall macht Azor deutlich, dass die Person Hilfe braucht. Er spricht von vier Dingen, die helfen können: 1) Abstand nehmen von Todsünden, im Fall des Konsums tierlicher Produkte wäre das beispielsweise von unmäßigem Konsum. 2) Dem Rat eines guten Menschen folgen, z. B. einem Arzt. 3) Über die Zweifel nachdenken. 4) Die falschen Dinge erkennen und somit die Skrupel beseitigen, denn einige Zweifel können sich durch Informationen, z. B. über die Tierhaltung, schon auflösen (Juan Azor 1600, lib. 2, cap. 20, q. 7). 
Eine Gewissensbildung bedarf also auch der persönlichen Arbeit. In dieser Eigeninitiative besteht das Wagnis. Es ist notwendig, sich aus der eigenen Komfortzone zu bewegen und mit Hilfe des kognitiven, des emotionalen und des Schrittes der Habitualisierung das eigene Gewissen weiterzubilden und danach zu handeln. Selbst im Zustand der Orientierungslosigkeit ist es durch die oben genannten Hilfsmittel möglich, Schritte zu setzen und sich damit weiter auf dem Weg des „Tutiorismus des Wagnisses“ zu bewegen.

\section{Schlussbemerkungen}

Eine Veränderung der Lebensgewohnheiten im Blick auf den Umgang mit tierlichen Produkten ist, wie deutlich wurde, in starkem Maße eine Herausforderung für die persönliche Gewissensbildung. Die Unterscheidung der verschiedenen „Gewissenslagen“ bei Juan Azor zeigt auf, wie auch heute handlungslähmende Gewissensempfindungen gedeutet, überwunden und aktive Gewissensentscheidungen vorbereitet werden können. Um die gegenwärtige Situation des vielfach ethisch nicht vertretbaren Konsums tierlicher Produkte zu verändern, ist die Arbeit jedes/jeder Einzelnen an sich selbst nötig. Hierfür ist nicht nur das Sammeln von Informationen über die einzelnen Konsumgüter erforderlich, sondern auch grundlegend die Beschäftigung mit dem eigenen Gewissen, eine Sensibilität für die eigenen Wahrnehmungen und Diskrepanzen zwischen Denken und Handeln sowie das Einüben von Gewohnheiten, die dem eigenen Gewissensurteil entsprechen. Im Sinne Karl Rahners könnte daher die Empfehlung Azors lauten, sich einen „Tutiorismus des Wagnisses“ anzueignen und sich im Sinne einer christlichen Verantwortung für die Schöpfung schrittweise auf den Weg zu einem rechten Gewissen zu begeben, d. h., der Stimme der Vernunft gegenüber falschen Gewohnheiten zunehmend mehr Raum zu verschaffen. In diesem Sinne können sich die praktisch orientierten Überlegungen aus der Anfangszeit der Moraltheologie als theologischem Fach auch für heutige tierethische Diskurse als stimulierend und fruchtbar erweisen.

\section{Literatur}

Ach, Johann S./Borchers, Dagmar (Hg.) 2018, Handbuch Tierethik, Stuttgart. Auer, Alfons 1971, Autonome Moral und christlicher Glaube, Düsseldorf. 
Azor, Juan 1600, Institutionum moralium. In quibus universae quaestiones ad conscientiam recte, aut prave factorum pertinentes, breviter tractantur. Pars prima, Rom.

Bundesministerium der Justiz und für Verbraucherschutz/Bundesamt für Justiz 1972, Tierschutzgesetz, in: http://www.gesetze-im-internet.de/tierschg/index.ht $\mathrm{ml}$ (abgerufen am 03.01.2020).

Ciraci, Joana 2019, Pelz? Nein, danke!, in: https://welttierschutz.org/pelz-nein-dank e/ (abgerufen am 19.11.2019).

Demmer, Klaus 1985, Deuten und Handeln. Grundlagen und Grundfragen der Fundamentalmoral, Freiburg etc.

Domingo, José L./Nadal, Martí 2017, Carcinogenicity of consumption of red meat and processed meat: A review of scientific news since the IARC decision, in: Food and Chemical Toxicology 105, 256-261.

Franziskus 2015, Laudato si'. Enzyklika über die Sorge für das gemeinsame Haus (24.05.2015).

Grabmann, Martin 1933, Die Geschichte der katholischen Theologie seit dem Ausgang der Väterzeit. Mit Benützung von M. J. Scheebens Grundriß dargestellt (Herders theologische Grundrisse), Freiburg i. Br.

Graça, João/Calheiros, Maria Manuela/Oliveira, Abílio 2016, Situating moral disengagement: Motivated reasoning in meat consumption and substitution, in: Personality and Individual Differences 90, 353-364.

Grill, Rupert 2019, „Willensschwäche“. Theologisch-ethische Neuverortung anhand der PSI-Theorie von Kuhl als Basis einer moralpsychologischen Perspektive, Habilitationsschrift Universität Wien.

Grimm, Herwig/Wild, Markus 2016, Tierethik zur Einführung, Hamburg.

Heinrich-Böll-Stiftung/Bund für Umwelt und Naturschutz Deutschland/Le Monde Diplomatique ${ }^{2} 2018$, Fleischatlas. Daten und Fakten über Tiere als Nahrungsmittel, Paderborn.

Henkel, Heinrich 1964, Einführung in die Rechtsphilosophie. Grundlagen des Rechts, München/Berlin.

Höfling, Gabriele 2016, Vegetarisches Essen ist Christenpflicht. Sozialethiker Kurt Remele über die Würde der Tiere, Bonn, in: https://www.katholisch.de/artikel/9 225-vegetarisches-essen-ist-christenpflicht (abgerufen am 19.11.2019).

Lintner, Martin M. 2017, Der Mensch und das liebe Vieh. Ethische Fragen im Umgang mit Tieren. Mit Beiträgen von Christoph J. Amor und Markus Moling, Innsbruck.

Lymbery, Philip 2015, Futtermittel: Viel Land für viel Vieh, in: https:/www.boell. $\mathrm{de} / \mathrm{de} / 2015 / 01 / 08 /$ futtermittel-viel-land-fuer-viel-vieh (abgerufen am 03.03.2020).

Mensink, Gert B. M./Lage Barbosa, Clarissa/Brettschneider, Anna-Kristin 2016, Verbreitung der vegetarischen Ernährungsweise in Deutschland, in: Journal of Health Monitoring 1 (2), 2-15.

Nethöfel, Wolfgang 1987, Moraltheologie nach dem Konzil. Personen, Programme, Positionen (Kirche und Konfession 26), Göttingen. 
Pollmer, Udo/Keckl, Georg/Alfs, Klaus 2015, Don't go Veggie! 75 Fakten zum vegetarischen Wahn, Stuttgart.

Rahner, Karl 1995, Selbstvollzug der Kirche. Ekklesiologische Grundlegung praktischer Theologie (Sämtliche Werke 19), Düsseldorf/Freiburg i. Br. etc.

Redaktion Fleischwirtschaft 2018, Zukunft der tierischen Erzeugung gestalten. Steigerung der Effizienz unter Beachtung des Umwelt-, Klima- und Ressourcenschutzes, in: Fleischwirtschaft 2, 30-31.

Regeniter, Hilde 2017, Fleischindustrie ist System struktureller Sünde. Tierrechtler sieht Kirche bei artgerechter Haltung in der Pflicht, in: https://www.domradio.d e/themen/ethik-und-moral/2017-02-14/tierrechtler-sieht-kirche-bei-artgerechter-h altung-der-pflicht (abgerufen am 19.11.2019).

Remele, Kurt 2016, Die Würde des Tieres ist unantastbar. Eine neue christliche Tierethik, Kevelaer.

Rosenberger, Michael 2015, Der Traum vom Frieden zwischen Mensch und Tier. Eine christliche Tierethik, München.

Schockenhoff, Eberhard 1990, Das umstrittene Gewissen. Eine theologische Grundlegung, Mainz.

Theiner, Johann 1970, Die Entwicklung der Moraltheologie zur eigenständigen Disziplin (Studien zur Geschichte der katholischen Moraltheologie 17), Regensburg.

Vogt, Markus 2019, Ethik des Wissens. Freiheit und Verantwortung der Wissenschaft in Zeiten des Klimawandels, München. 
